\title{
Anastomotic leakage after curative rectal cancer resection has no impact on long-term survival: a propensity score analysis
}

\author{
Sabrina M. Ebinger ${ }^{1} \cdot$ René Warschkow $^{1,2}$ • Ignazio Tarantino ${ }^{3} \cdot$ Bruno M. Schmied $^{1}$. \\ Lukas Marti ${ }^{1,4}$
}

Accepted: 26 July 2015 /Published online: 6 August 2015

(C) Springer-Verlag Berlin Heidelberg 2015

\begin{abstract}
Purpose Anastomotic leakage (AL) is a severe and frequent complication of rectal cancer resection, with an incidence rate of approximately $9 \%$. Although the impact of AL on morbidity and short-term mortality has been established, the literature is contradictory regarding its influence on long-term, cancerspecific survival. The present investigation assessed the longterm survival of 584 patients with stage I-III rectal cancer. Methods The 10-year overall survival and cancer-specific survival were analyzed in 584 patients from a single tertiary center. All patients had undergone curative rectal cancer resection between 1991 and 2010. Patients with and without AL were compared using both a multivariate Cox hazards model and propensity score analysis.

Results A total of 64 patients developed AL (11.0\%, $95 \%$ confidence interval $(\mathrm{CI})=8.7$ to $13.8 \%$ ). The median followup was 5.2 years for all patients; and 7.4 years for patients still alive at the end of the investigated period. AL did persistently not impair cancer-specific survival based on unadjusted Cox regression (hazard ratio of death $(\mathrm{HR})=1.27,95 \% \mathrm{CI}=0.65$ to 2.48, $P=0.489)$; risk-adjusted Cox regression $(\mathrm{HR}=1.10$,
\end{abstract}

Lukas Marti

Lukas.Marti@kssg.ch

1 Department of Surgery, Cantonal Hospital of St. Gallen, 9007 St. Gallen, Switzerland

2 Institute of Medical Biometry and Informatics, University of Heidelberg, 69120 Heidelberg, Germany

3 Department of Surgery, University of Heidelberg, 69120 Heidelberg, Germany

4 Department of Surgery, University-Hospital Mannheim, Medical Faculty Mannheim, University of Heidelberg, 68167 Mannheim, Germany
$95 \% \mathrm{CI}=0.54$ to $2.20, P=0.799)$; and propensity score matching $(\mathrm{HR}=1.18,95 \% \mathrm{CI}=0.57$ to $2.43, P=0.660)$. Conclusions Based on the present propensity score analysis, the oncologic outcomes in patients undergoing curative rectal cancer resections were not impaired by the development of anastomotic leakage.

Keywords Rectal cancer resection - Anastomotic leackage · Overall survival $\cdot$ Cancer-specific survival $\cdot$ Propensity score matching

\section{Introduction}

Oncologic outcomes after curative rectal cancer resections are evidently influenced by several tumor- and patient-specific characteristics, such as a higher pT stage, a worse tumor differentiation, and an older age [1-3], most of which are not controllable. Whether anastomotic leakage (AL), a major and feared complication of rectal surgery, can be added to these determinants remains a matter of debate. Meanwhile, it has been shown that $\mathrm{AL}$ is one of the major causes of morbidity and short-term mortality resulting from rectal cancer resections $[4,5]$. However, studies are contradictory with respect to long-term and cancer-specific survival. A recent multicenter study pooling data from five randomized clinical trials found an impaired overall survival after AL, whereas the cancerspecific survival was not significantly reduced [6]. In contrast to these findings, a recent meta-analysis of 21 trials concluded that local recurrence and cancer-specific survival were both negatively associated with AL [7]. One reason for this inconsistency may be that retrospective analysis, unlike randomized clinical trials (RCTs), carries the risk of selection bias (sampling bias). In the present case, the two groups of patients with and without AL could have exhibited different distributions of 
confounding variables (omitted variable bias). In other words, the variables that are risk factors for AL, including preexisting vascular disease, intra- and postoperative blood substitution, and more advanced UICC stage [8], may also be risk factors for diminished oncologic outcome.

When adjusting for these potential confounders, propensity score matching (PSM) is a better statistical method than the typically applied multivariate Cox proportional hazards mod$\mathrm{el}$, as the former can reduce selection bias by simulating randomization. This approach creates a sample of patients with AL that is comparable, with respect to all the observed covariates, to a sample of patients without $\mathrm{AL}$.

Because the reported incidence of AL is as high as $3-33 \%$ [7], it is of utmost importance to clarify the correlation between $\mathrm{AL}$ and oncologic outcomes. A positive correlation would influence the follow-up and treatment decisions made by clinicians. The aim of the present retrospective study was to answer this question.

\section{Patients and methods}

The present retrospective study was based on the colorectal database of the authors' institution, one of the largest tertiary care centers in Switzerland. Using this valuable database, our research group previously assessed risk factors for $\mathrm{AL}$, as well as the impact of the preoperative carcinoembryonic antigen (CEA) level and blood substitution on survival after rectal cancer resection [8]. Overall, 918 patients undergoing open rectal cancer resection between January 1991 and December 2010 were identified. Patients who received no anastomosis due to undergoing Hartman's or abdominoperineal resection $(N=88)$, dying intraoperatively $(N=1)$, or undergoing transanal resection $(N=25)$ were excluded, as were patients with distant metastases at the time of operation $(N=157)$, patients not undergoing elective surgery $(N=33)$, patients with incomplete tumor resections $(N=7)$, and patients lost to follow-up $(N=12)$. In-hospital mortality occurred in 11 of the remaining 595 patients $(1.8 \%, 95 \%$ confidence interval $[\mathrm{CI}]=1.0$ to $3.3 \%$ ), who were also excluded. Two of the 66 patients with AL, compared with 9 of the 529 patients without anastomotic leakage, died in-hospital during the study period ( 3.0 vs. $1.7 \%, P=0.450$ ). A total of 584 patients were ultimately enrolled for further analyses (Fig. 1).

\section{Data collection and definitions}

Data on the patients' characteristics and outcomes were ascertained retrospectively from medical charts. The follow-up for the first five postoperative years was performed according to the national guidelines for colorectal cancer. Thereafter, the patients and their general practitioners were contacted by mail and telephone to obtain information regarding their survival status. If

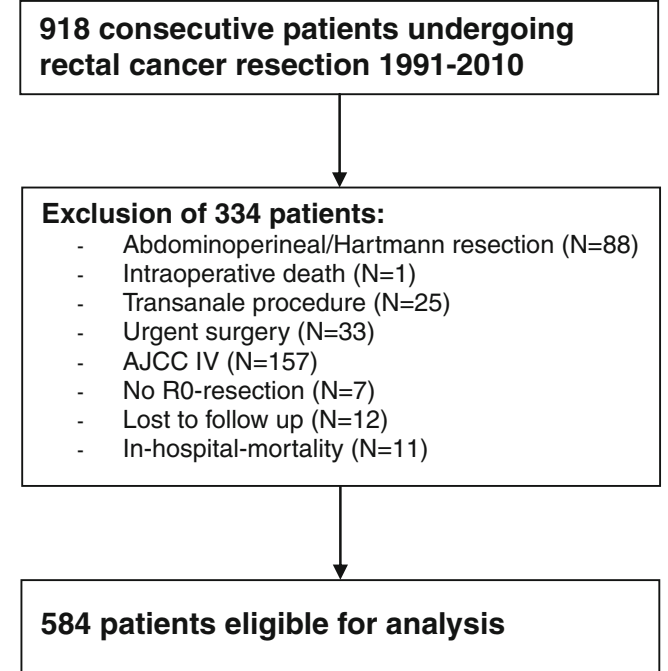

Fig. 1 Patient selection: depicts the process of patient selection and exclusion criteria

no information was available, the local residents' registration offices were contacted to achieve the most complete follow-up possible. Follow-up was completed in 2012.

Tumor height was defined as the distance between the tumor and the anal verge and was calculated based on the results of rigid rectosigmoidoscopy, endorectal sonography, magnetic resonance imaging (MRI) scans, and colonoscopy. All operations were performed or supervised by experienced colorectal surgeons. AL was defined as the presence of a pelvic abscess near the anastomosis; proof of AL through an intestinal wall defect by rectal examination, sigmoidoscopy, extravasation of an endoluminally administered water-soluble contrast agent determined by radiography or computed tomography; or proof of AL upon re-operation. Neoadjuvant radiotherapy was administered according to an interdisciplinary tumor board decision in patients classified as $\mathrm{uT} 3 / 4$ or $\mathrm{uN}+$ based on preoperative staging. Adjuvant chemotherapy was routinely administered to patients with node-positive disease according to the pre- and postoperative staging [9].

\section{Statistical analyses}

Statistical analyses were performed using the R statistical software (www.r-project.org). A two-sided $P$ value $<0.05$ was considered statistically significant. Continuous data are expressed as means \pm standard deviations. Chi-square statistics, $t$ tests and Mann-Whitney $U$ tests were used as appropriate.

The overall and cancer-specific survival rates were defined as the main outcome. Regarding cancer-specific survival, patients with an unknown cause of death $(N=55)$ were excluded. The risk factors included in the descriptive and multivariate analyses were age, gender, body mass index, American Society of Anesthesiologists (ASA) classification, coronary arterial occlusive disease, preoperatively elevated $(5 \mathrm{ng} / \mathrm{ml}$ or 
higher) carcinoembryonic antigen (CEA) level [10], tumor distance from the anal verge, American Joint Committee on Cancer (AJCC) stage, neoadjuvant therapy, total vs. partial mesorectal excision, operative time, diverting stoma, epidural analgesia, intraoperative blood loss, lowest perioperative hemoglobin level, and perioperative blood transfusions (the latter two of which were assessed until postoperative day 7 and only before diagnosis of $\mathrm{AL}$ ). Missing data were imputed using the random survival forest method [11].

First, all variables in the risk set were compared between patients with and without AL based on univariate analyses and multivariate logistic regression. The same covariates as those used in the risk set, including $\mathrm{AL}$, were then assessed as putative prognostic factors for overall and cancer-specific survival in unadjusted and riskadjusted Cox regressions. Additional backward-variable selection procedures derived from the full Cox regression models were performed based on Akaike's information criterion. Moreover, a propensity score analysis was performed as a superior and more refined statistical method of adjusting for potential baseline confounding variables [12-14]. We used the "Matching" R package to perform a bipartite weighting propensity score analysis $[15,16]$. The baseline risk profiles of the matched patients were compared to assure that no major differences in baseline patient characteristics persisted. The prognostic value of $\mathrm{AL}$ for overall and disease-free survival was finally assessed in a stratified Cox regression analysis after applying the subclasses and weights obtained by the propensity score analysis.

\section{Results}

\section{Patient characteristics and risk factors for anastomotic leakage}

A total of 584 patients were included in the analysis. Two groups were compared, including 64 patients with $\mathrm{AL}$ $(11.0 \%, 95 \% \mathrm{CI}=8.7$ to $13.8 \%)$ and 520 patients who did not develop AL. A diagnosis of AL was made between postoperative days 3 and 34 , at a median of 8 days postoperatively. In 33 patients (5.7\%), a re-operation was required, whereas in the remaining 31 patients $(5.3 \%)$, the AL could be managed without re-operation.

The median follow-up was 5.2 years (range 0.2 to 21.2 years) for all 584 patients and 7.4 years for the 401 patients who were alive at the end of the follow-up. Table 1 summarizes the characteristics of patients with and without AL. Significant differences were observed in perioperative hemoglobin levels, operative times, and perioperative blood transfusions. In the multivariate logistic regression analysis, operative time and perioperative blood transfusions were independent, statistically significant predictors of developing AL (Table 1).

\section{Short-term outcomes and recurrences}

Regarding the short-term outcomes, patients with AL had a

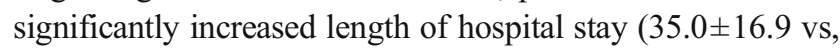
$20.6 \pm 11.1$ days, $P<0.001$ ). Only 12 of the 64 patients with AL (18.8\%), compared with 199 of the 520 patients without $\mathrm{AL}(38.3 \%)$, underwent adjuvant therapy $(P=0.002)$.

In $101(17.3 \%)$ patients, a recurrence of the rectal cancer was observed. Among these, 31 (5.3\%) patients suffered a local recurrence, $50(8.6 \%)$ patients had metachronous liver metastases and $42(7.2 \%)$ patients had pulmonary metastases. Local recurrence was recorded in 4 (6.2\%) patients with AL compared to $27(5.2 \%)$ patients without AL $(P=0.722)$. Hepatic recurrence was recorded in $6(9.4 \%)$ patients with $\mathrm{AL}$ compared to $44(8.5 \%)$ patients without AL $(P=0.805)$. Pulmonary recurrence was recorded in $3(4.7 \%)$ patients with $\mathrm{AL}$ compared to $39(7.5 \%)$ patients without AL $(P=0.411)$. The 5 -year cumulative incidence of local recurrence in patients with $\mathrm{AL}$ was $6.9 \%(95 \% \mathrm{CI}=0.1$ to $13.3 \%)$ compared to $5.4 \%(95 \% \mathrm{CI}=3.3$ to $7.5 \%)$ in patients without $\mathrm{AL}(P=$ 0.656 in Cox regression).

\section{Assessment of anastomotic leakage as a prognostic factor for survival}

In the unadjusted Cox proportional hazards regression, AL did not significantly influence overall survival (hazard ratio of death $=1.00,95 \% \mathrm{CI}=0.63$ to $1.59, P=0.999)$ or cancerspecific survival (hazard ratio of death $=1.27,95 \% \mathrm{CI}=0.65$ to $2.48, P=0.489$ ) (Table 2). The 5- and 10-year overall survival rates of patients with $\mathrm{AL}$ were $80.1 \%(95 \% \mathrm{CI}=70.1$ to $91.5 \%$ ) and $62.6 \%$ (95\% CI=50.4 to $77.8 \%)$, respectively, compared with $78.5 \%(95 \% \mathrm{CI}=74.8$ to $82.3 \%)$ and $62.7 \%$ (95\% CI=57.9 to $67.9 \%$ ), respectively, in patients without AL (Fig. 2, left upper panel). The 5- and 10-year cancer-specific survival rates of patients with AL were $89.6 \%(95 \%$ $\mathrm{CI}=81.3$ to $98.7 \%)$ and $76.2 \%(95 \% \mathrm{CI}=64.2$ to $90.5 \%)$, respectively, compared with $88.6 \%(95 \% \mathrm{CI}=85.6$ to $91.8 \%)$ and $82.2 \%(95 \% \mathrm{CI}=78.1$ to $86.6 \%)$, respectively, in patients without AL (Fig. 2, right upper panel). After adjusting for potential confounding factors in the riskadjusted Cox regression analyses, $\mathrm{AL}$ was confirmed to have no statistically significant influence on overall survival (hazard ratio of death $=0.91,95 \% \mathrm{CI}=0.56$ to $1.47, P=0.701$ ) or cancer-specific survival (hazard ratio of death $=1.10,95 \%$ $\mathrm{CI}=0.54$ to $2.20, P=0.799$ ). Following backward-variable selection procedures, $\mathrm{AL}$ was not found to be a significant prognostic factor for overall or cancer-specific survival. 


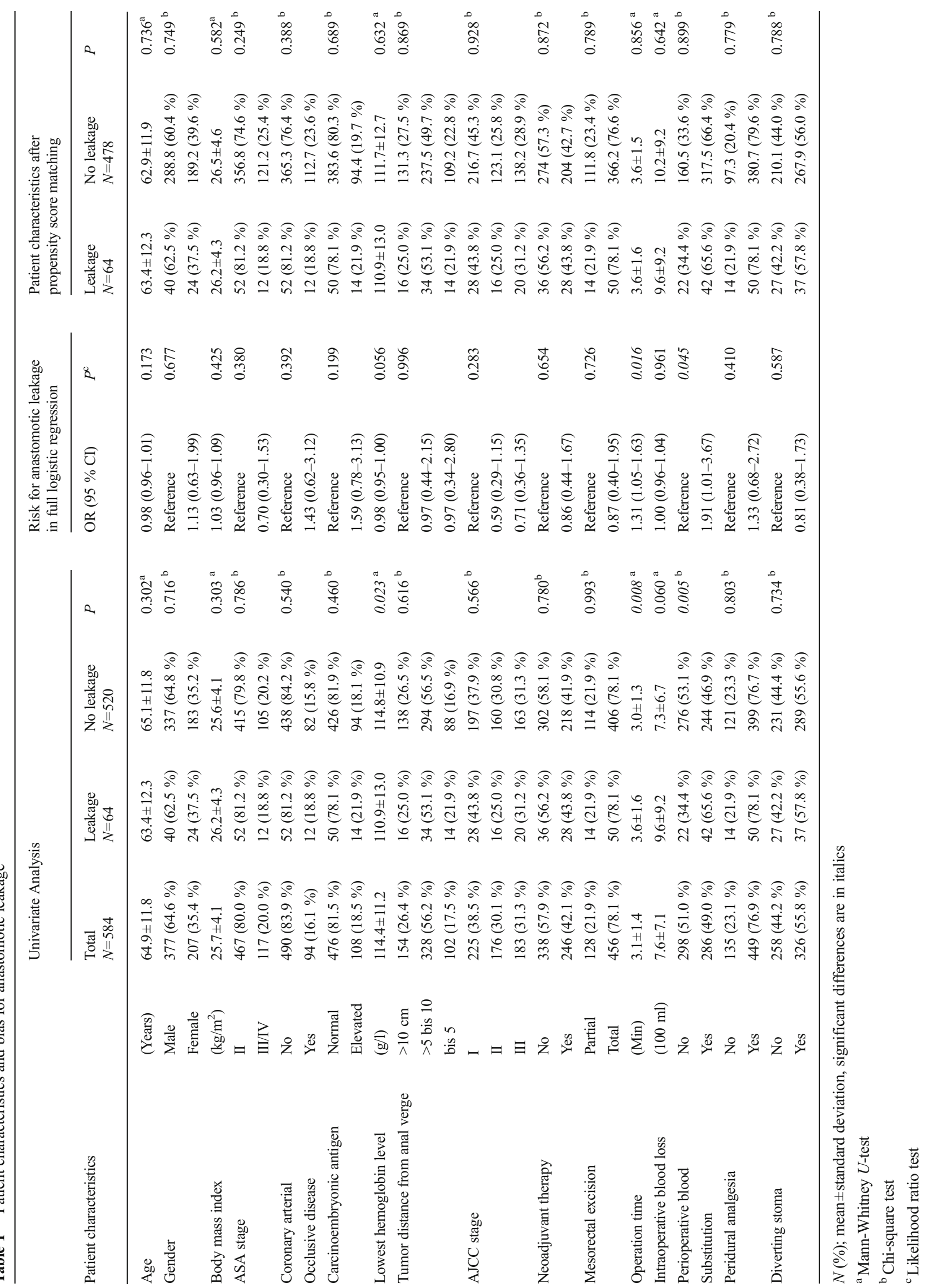




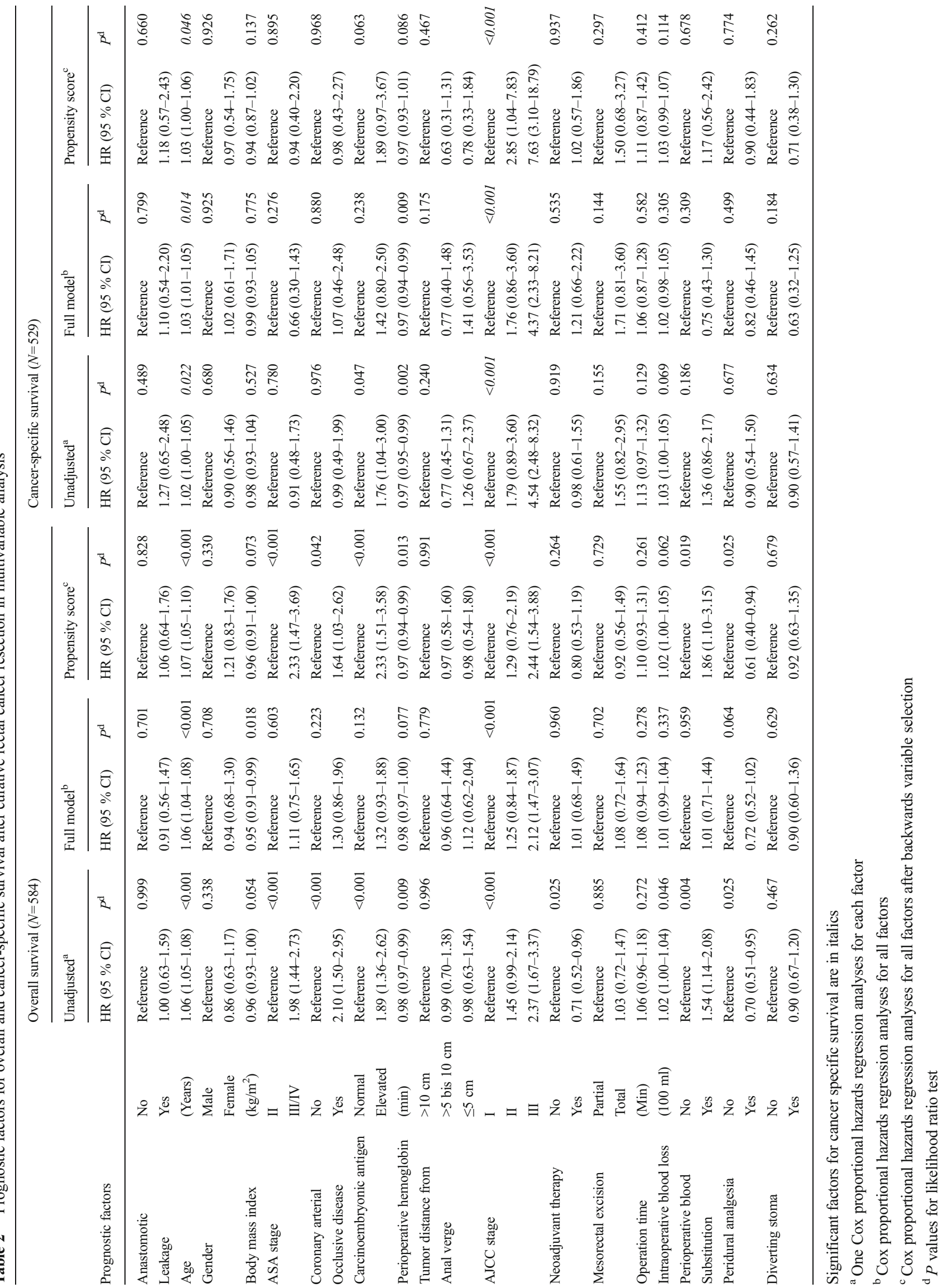



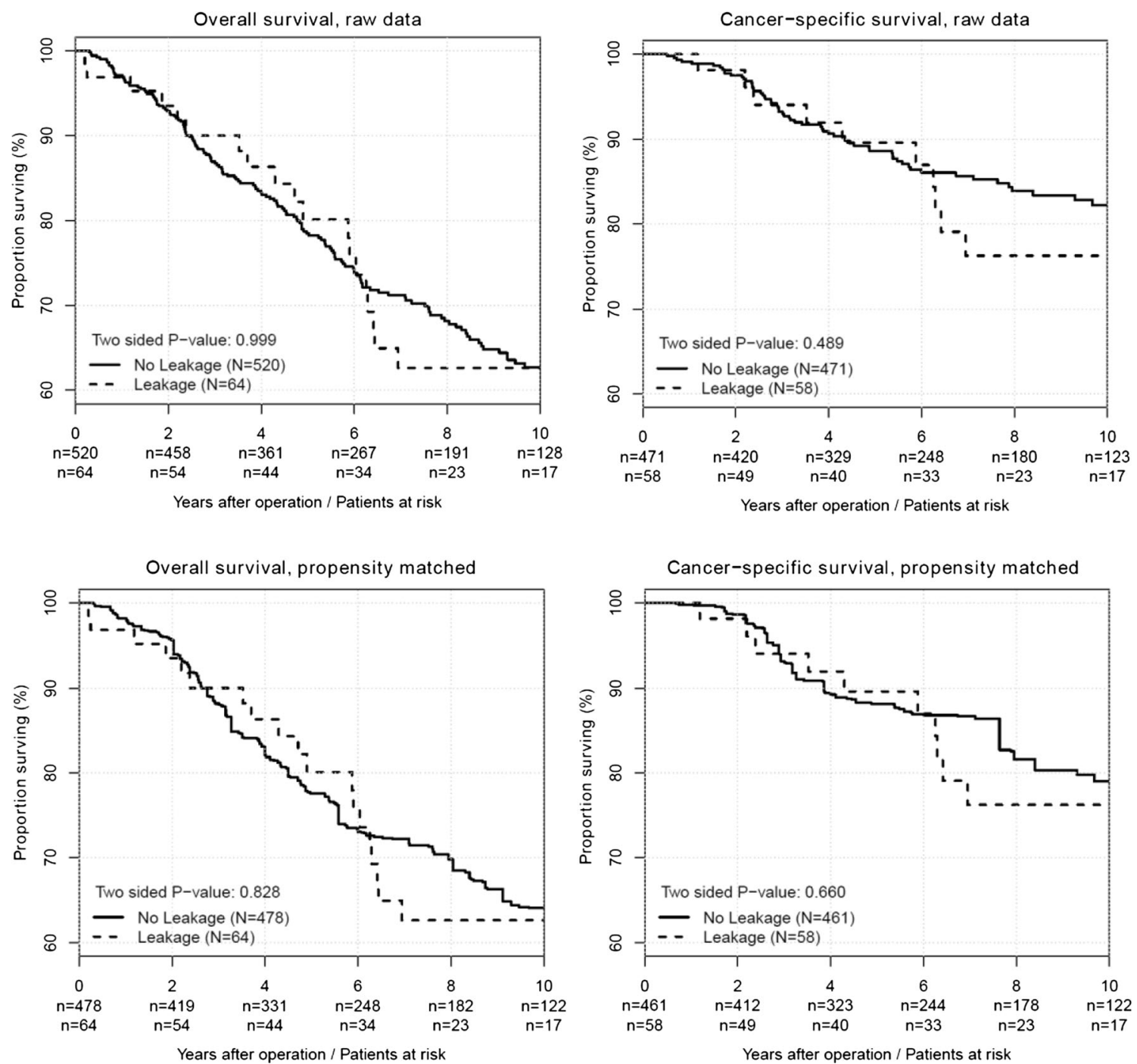

Fig. 2 Overall and cancer-specific survival: Kaplan-Meier curve for overall and cancer-specific survival in unadjusted (left and right upper panel) and propensity score adjusted analysis (left and right lower panel). The number of rectal cancer patients at risk is given below each plot

\section{Adjusting for patient characteristics with propensity score matching}

The propensity score in patients who developed AL was 0.16 \pm 0.09 , compared with $0.11 \pm 0.07$ in those who did not develop AL $(P<0.001)$, indicating a significant bias in the patient characteristics. When performing the propensity score matching procedure, 42 patients without AL had to be excluded because their characteristics could not be matched to those of patients from the other group. Thus, the propensity scorebased analysis was based on 542 patients. The propensity scores derived from the analysis were virtually the same in patients who did and did not develop AL (0.16 \pm 0.09 vs. 0.16 $\pm 0.09, P=0.986)$. Figure 3 displays the distribution of propensity scores and the relative weights of the patients after the matching procedure.

After adjusting the data according to the propensity score analysis, AL still had no significant impact on overall survival (hazard ratio of death $=1.06,95 \% \mathrm{CI}=0.64$ to $1.76, P=0.828$ ) or cancer-specific survival (hazard ratio of death $=1.18,95 \%$ $\mathrm{CI}=0.57$ to $2.43, P=0.660$ ) (Table 2 ). Additionally, the survival curves did not differ significantly after propensity score matching (Fig. 2, left and right lower panels).

\section{Discussion}

The key result of the present study is that $\mathrm{AL}$ after curative rectal cancer resection does not impact long-term overall or cancerspecific survival. These findings are supported by both a conventional multivariate Cox proportional hazards model and by the data accrued after propensity score matching, even though the patient characteristics were previously significantly biased. Intraoperative blood loss, perioperative blood substitution, and a longer operating time were risk factors for developing AL but, consistent with previous studies [17], were not associated with 
Distribution of Propensity Scores

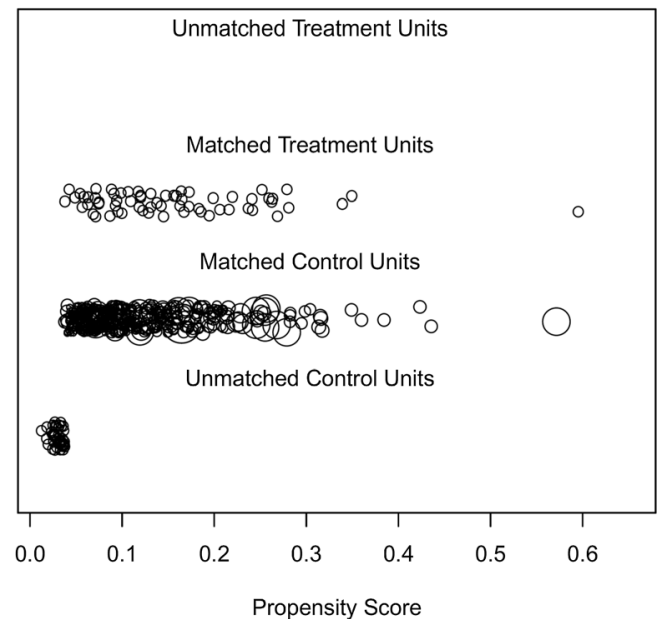

Fig. 3 Distribution of propensity scores before and after propensity score analysis: Each circle presents one patient. The distribution of the propensity scores for patients with and without anastomotic leakage ("treatment units" and "control units") who could be matched is shown. The propensity scores for patients who could not be matched because their characteristics could not be matched with patients from the other group are also shown. The size of the circle for matched patients without anastomotic leakage ("control units") represents the weight obtained by the propensity score matching procedure

worse long-term outcomes. Among the analyzed variables, only a higher AJCC stage was identified as a clear and significant risk factor for worse cancer-specific survival.

The present study added new valuable evidence to the present research question by using propensity score matching to mimic randomization. Additionally, our study included a comparatively large number of patients and a rather long and complete follow-up. The observed results align with those of other investigations that also found that $\mathrm{AL}$ was not a risk factor for disease-specific survival. In a prospective analysis of 2480 patients, den Dulk et al. concluded that AL reduced overall but not cancer-specific survival. Another result of their analysis was that AL did not influence local recurrence [6]. Smith et al. performed a retrospective analysis of stage IV rectal cancer patients in 2013, and again, AL was not shown to be negatively associated with local recurrence, disease-free survival, or overall survival [18].

A few studies, however, have yielded contradictory results $[19,20,7,21-23]$. In their recent meta-analysis of predominantly retrospective trials, Mirnezami et al. [7] concluded that AL has a negative prognostic impact on local recurrence and leads to a reduced long-term cancer-specific survival. There are several explanations for these contradictory results. Apart from the aforementioned statistical reasons, such as selection bias and the influence of putative confounders on retrospective trials, some studies have been limited to certain severities of AL. Accordingly, in the metaanalysis by Mirnezami et al., the incidence rate of AL varied remarkably between 0.5 and $30 \%$. Furthermore, their review included studies between 1965 and 2009, which may have biased the outcomes due to the changing diagnostic and therapeutic treatment strategies during this time period. If the treatment and diagnosis of $\mathrm{AL}$ are not sufficient, delays may lead to postponed or canceled adjuvant therapy, which could also explain the worse survival. In a retrospective analysis of the success of fibrin glue in the treatment of $\mathrm{AL}, \mathrm{Kim}$ et al. [20] found that $\mathrm{AL}$ is an independent prognostic factor for disease-free survival. They also applied propensity score matching. However, the authors stated that they could not thoroughly investigate the correlations between AL, fibrin glue, and oncologic outcomes due to the small number of events. Furthermore, this study only included clinically, but not radiographically, proven AL.

We acknowledge the limitations of the present study. First, this is a cohort study and not a prospective trial. Thus, the inherent disadvantages of our design may represent sources of bias, e.g., recall bias, information bias, and selection bias. To minimize this risk of bias, we performed additional propensity score matching. Propensity score matching, however, can only adjust for factors that have been appropriately measured, whereas randomization can resolve bias from both measured and unmeasured (respectively unknown) factors or those that interact in complex manners.

For the present patient cohort data about the quality of mesorectal excision and the circumferential resection margins are lacking. These variables were recently proven as relevant prognostic factors [24]. However, microscopic involvement of any margin of the specimen (R1) was assessed by the pathologist and detected rather low. Patients with any microscopic involvement of any margin of the specimen were excluded from the analysis. Therefore and because of the long follow-up time of the present investigation, the results found are still valuable. Moreover, these factors would be, if different, expected to be worse in patients with $\mathrm{AL}$ and thus are not to be expected to alter the results of this analysis.

Other variables that were not tested but may have influenced the outcome may include the volume of the clinic/surgeon, type of adjuvant therapy, specific genetic mutations, drainage, or a higher socioeconomic status $[25,26]$. Nevertheless, propensity score matching is a promising addition to conventional methods [27]. Based on the present research question, a cohort study is probably the most appropriate study design, as an extremely large population would be required in the setting of a RCT for a potentially low number of events (AL, recurrence, death).

A second limitation of our analysis is the long investigation period of almost 20 years. Diagnostic and therapeutic strategies may have changed during this period, which could be a potential source of additional bias.

We conclude that, according to our data, the long-term overall survival and cancer-specific survival after curative resection of rectal cancer are not impaired by AL. However, as 
contradictory results remain in the literature regarding this issue, no conclusive guidelines can be proposed. Therefore, every possible precaution should be undertaken to prevent AL. Postoperative surveillance should be standardized to detect [28] and adequately treat AL, such that any severe negative impacts on a patient's short-term outcome can be prevented and any necessary adjuvant therapy is not postponed. A close-meshed oncologic follow-up for patients after AL should be conducted until its influence on local recurrence and survival is determined with certainty.

Conflict of interest The authors declare that they have no competing interests.

Compliance with ethical standards For this type of study formal consent is not required. However all patients agreed that their data may be used for scientific analyses on a general consenting form, when they entered the hospital. These proceedings were generally approved by the local ethic board.

\section{References}

1. Kim SH, Bae KB, Kim JM, Shin JH, An MS, Ha TG, Ryu SM, Kim KH, Kim TH, Choi CS, Shin JY, Oh M, Baek SH, Hong KH (2012) Oncologic outcomes and risk factors for recurrence after tumorspecific mesorectal excision of rectal cancer: 782 cases. J Korean Soc Coloproctol 28(2):100-107. doi:10.3393/jksc.2012.28.2.100

2. Chen ZH, Song XM, Chen SC, Li MZ, Li XX, Zhan WH, He YL (2012) Risk factors for adverse outcome in low rectal cancer. World J Gastroenterol 18(1):64-69. doi:10.3748/wjg.v18.i1.64

3. Reshef A, Lavery I, Kiran RP (2012) Factors associated with oncologic outcomes after abdominoperineal resection compared with restorative resection for low rectal cancer: patient- and tumorrelated or technical factors only? Dis Colon Rectum 55(1):51-58. doi:10.1097/DCR.0b013e3182351c1f

4. Karanjia ND, Corder AP, Bearn P, Heald RJ (1994) Leakage from stapled low anastomosis after total mesorectal excision for carcinoma of the rectum. Br J Surg 81(8):1224-1226

5. Snijders HS, Wouters MW, van Leersum NJ, Kolfschoten NE, Henneman D, de Vries AC, Tollenaar RA, Bonsing BA (2012) Meta-analysis of the risk for anastomotic leakage, the postoperative mortality caused by leakage in relation to the overall postoperative mortality. Eur J Surg Oncol 38(11):1013-1019. doi:10.1016/j.ejso. 2012.07.111

6. den Dulk M, Marijnen CA, Collette L, Putter H, Pahlman L, Folkesson J, Bosset JF, Rodel C, Bujko K, van de Velde CJ (2009) Multicentre analysis of oncological and survival outcomes following anastomotic leakage after rectal cancer surgery. Br J Surg 96(9):1066-1075. doi:10.1002/bjs.6694

7. Mirnezami A, Mirnezami R, Chandrakumaran K, Sasapu K, Sagar P, Finan P (2011) Increased local recurrence and reduced survival from colorectal cancer following anastomotic leak: systematic review and meta-analysis. Ann Surg 253(5):890-899. doi:10.1097/ SLA.0b013e3182128929

8. Warschkow R, Steffen T, Thierbach J, Bruckner T, Lange J, Tarantino I (2011) Risk factors for anastomotic leakage after rectal cancer resection and reconstruction with colorectostomy. A retrospective study with bootstrap analysis. Ann Surg Oncol 18(10): 2772-2782. doi:10.1245/s10434-011-1696-1
9. Sauer R, Becker H, Hohenberger W, Rodel C, Wittekind C, Fietkau R, Martus P, Tschmelitsch J, Hager E, Hess CF, Karstens JH, Liersch T, Schmidberger H, Raab R (2004) Preoperative versus postoperative chemoradiotherapy for rectal cancer. N Engl J Med 351(17):1731-1740. doi:10.1056/NEJMoa040694

10. Locker GY, Hamilton S, Harris J, Jessup JM, Kemeny N, Macdonald JS, Somerfield MR, Hayes DF, Bast RC Jr (2006) ASCO 2006 update of recommendations for the use of tumor markers in gastrointestinal cancer. J Clin Oncol 24(33):53135327. doi:10.1200/jco.2006.08.2644

11. Ishwaran H, Kogalur UB, Blackstone EH, Lauer MS (2008) Random survival forests. Ann Appl Stat 841-860. doi:10.1214/08-AOAS169

12. Joffe MM, Rosenbaum PR (1999) Invited commentary: propensity scores. Am J Epidemiol 150(4):327-333

13. Rubin DB (1997) Estimating causal effects from large data sets using propensity scores. Ann Intern Med 127(8 Pt 2):757-763

14. Rosenbaum PR (1987) Model-based direct adjustment. J Am Stat Assoc 82(398):387-394. doi:10.1080/01621459.1987.10478441

15. Hansen BB, Klopfer SO (2006) Optimal full matching and related designs via network flows. J Comput Graph Stat 15:609-627

16. Sekhon JS (2011) Multivariate and propensity score matching software with automated balance optimization: the matching package for R. J Stat Softw 42:1-52

17. Warschkow R, Guller U, Koberle D, Muller SA, Steffen T, Thurnheer M, Schmied BM, Tarantino I (2014) Perioperative blood transfusions do not impact overall and disease-free survival after curative rectal cancer resection: a propensity score analysis. Ann Surg 259(1):131-138. doi:10.1097/SLA.0b013e318287ab4d

18. Smith JD, Butte JM, Weiser MR, D'Angelica MI, Paty PB, Temple LK, Guillem JG, Jarnagin WR, Nash GM (2013) Anastomotic leak following low anterior resection in stage IV rectal cancer is associated with poor survival. Ann Surg Oncol 20(8):2641-2646. doi:10. 1245/s10434-012-2854-9

19. Bertelsen CA, Andreasen AH, Jorgensen T, Harling H (2010) Anastomotic leakage after curative anterior resection for rectal cancer: short and long-term outcome. Colorectal Dis 12 (7 Online): e76-81. doi:10.1111/j.1463-1318.2009.01935.x

20. Kim HJ, Huh JW, Kim HR, Kim YJ (2014) Oncologic impact of anastomotic leakage in rectal cancer surgery according to the use of fibrin glue: case-control study using propensity score matching method. Am J Surg 207(6):840-846. doi:10.1016/j.amjsurg.2013.07.047

21. Eberhardt JM, Kiran RP, Lavery IC (2009) The impact of anastomotic leak and intra-abdominal abscess on cancer-related outcomes after resection for colorectal cancer: a case control study. Dis Colon Rectum 52(3):380-386. doi:10.1007/DCR.0b013e31819ad488

22. Gong JP, Yang L, Huang XE, Sun BC, Zhou JN, Yu DS, Zhou X, Li DZ, Guan X, Wang DF (2014) Outcomes based on risk assessment of anastomotic leakage after rectal cancer surgery. Asian Pac J Cancer Prev 15(2):707-712

23. Kulu Y, Tarantio I, Warschkow R, Kny S, Schneider M, Schmied BM, Buchler MW, Ulrich A (2015) Anastomotic leakage is associated with impaired overall and disease-free survival after curative rectal cancer resection: a propensity score analysis. Ann Surg Oncol 22(6):2059-2067. doi:10.1245/s10434-014-4187-3

24. Quirke P, Steele R, Monson J, Grieve R, Khanna S, Couture J, O'Callaghan C, Myint AS, Bessell E, Thompson LC, Parmar M, Stephens RJ, Sebag-Montefiore D (2009) Effect of the plane of surgery achieved on local recurrence in patients with operable rectal cancer: a prospective study using data from the MRC CR07 and NCIC-CTG CO16 randomised clinical trial. Lancet 373(9666): 821-828. doi:10.1016/s0140-6736(09)60485-2

25. Dik VK, Aarts MJ, Van Grevenstein WM, Koopman M, Van Oijen MG, Lemmens VE, Siersema PD (2014) Association between socioeconomic status, surgical treatment and mortality in patients with colorectal cancer. Br J Surg 101(9):1173-1182. doi:10.1002/bjs.9555 
26. Jung SH, Yu CS, Choi PW, Kim DD, Park IJ, Kim HC, Kim JC (2008) Risk factors and oncologic impact of anastomotic leakage after rectal cancer surgery. Dis Colon Rectum 51(6):902-908. doi: 10.1007/s10350-008-9272-x

27. Sturmer T, Joshi M, Glynn RJ, Avorn J, Rothman KJ, Schneeweiss S (2006) A review of the application of propensity score methods yielded increasing use, advantages in specific settings, but not substantially different estimates compared with conventional multivariable methods. J Clin Epidemiol 59(5):437-447. doi:10.1016/j. jclinepi.2005.07.004

28. Singh PP, Zeng IS, Srinivasa S, Lemanu DP, Connolly AB, Hill AG (2014) Systematic review and meta-analysis of use of serum Creactive protein levels to predict anastomotic leak after colorectal surgery. Br J Surg 101(4):339-346. doi:10.1002/bjs.9354 